\title{
INFLUÊNCIA DA ADIÇÃO DE FÓSFORO NO ÍNDICE DE COMPRESSÃO E PARÂMETROS DE CONSISTÊ NCIA DE UM LATOSSOLO VERMELHO-ESCURO(1)
}

\author{
R. B. SILVA(2), J . M. LIMA(3), M. S. DIAS J UNIOR ${ }^{(3)}$ \& F. A. M. SILVA(2)
}

\begin{abstract}
RESUMO
A adsorção de fosfato pelo solo pode causar a dispersão de partículas, alterando sua porosi dade e a relação solo-água, especialmente em solos oxídicos. Portanto, parâmetros do solo variáveis com o conteúdo de água, como os li mites de consistência e índices de compressão, podem, indiretamente, ser influenciados pela adsorção desse íon. Este trabalho teve como objetivo avaliar o efeito da adsorção de fosfato no índice de compressão e nos limites de consistência de um Latossolo Vermelho-E scuro da microrregião dos Campos da Mantiqueira (MG), Brasil. Amostras do solo foram coletadas nas camadas de 0 $0,03 \mathrm{~m}$ e $0,27-0,30 \mathrm{~m}$. Metade das amostras recebeu fosfato durante a fase de pré-umedecimento no laboratório, com $\mathbf{P}$ suficiente para atingir a capacidade de adsorção. Foram determinados os limites de consistência e o índice de compressão, este representado pela inclinação da reta de compressão virgem da curva de compressão. Os valores do índice de compressão foram mais elevados para a camada superficial, tanto na condi ção com $P$ quanto na sem $P$, significando maior predisposição à compactação. Os limites de consistência e o índice de compressão foram mais elevados para condição com P. E mbora o teor de água óti mo para a mai or resposta à compactação esteja dentro da faixa de plasticidade na camada superficial, o que significa menor risco de compactação com o solo preparado na faixa de friável, o solo ainda seria susceptível à compactação, uma vez que esses valores estão na faixa de friabilidade para a camada de 0,270,30 m; camada esta que coincide com a profundidade de corte de alguns dos implementos de preparo do solo.
\end{abstract}

Termos de indexação: plasticidade, friabilidade, compactação do solo, dispersão de partículas, adsorção de fósforo.

\footnotetext{
(1) Parte da Tese de Mestrado do primeiro autor, apresentada à Universidade Federal de Lavras - UFLA. Projeto financiado pela CAPES. Recebido para publicação em junho de 2000 e aprovado em novembro de 2000.

(2) Engenheiro-Agrônomo, Pós-Graduando do Departamento de Ciência do Solo, Universidade Federal de Lavras - UFLA. Caixa Postal 37, CEP 37200-000 Lavras (MG). Bolsista da CAPES. E-mail: rbsilva@ufla.br

(3) Professor do Departamento de Ciência do Solo, UFLA. Bolsista do CNPq.
} 


\title{
SUMMARY: EFFECT OF PHOSPHATE SORPTION ON THE COMPRESSION INDEX AND CONSISTENCE PARAMETERS OF OXISOLS
}

\begin{abstract}
Phosphatesorption can causeparticl edispersion, changing soil porosity, and affecting soil-water relationships, mainly in oxidic soils. Therefore, it can also affect water-related parameters, such as consistence limits and response to soil compaction. The objective of this study was to test the effect of phosphatesorption on consistencelimits and compressi on indexes of undisturbed samples from 0-0.03 and 0.27-0.3 m-layers of a Dark Red Latosol from Campos das Vertentes, Minas Gerais, Brazil. Phosphate was added to half of the samples, during the prewetting phase of the compressibility test, in order to reach their maximum sorption capacity. Compression index represents the slope of the virgin part of the compression curve The surface layer showed higher compression indexes for both $\mathrm{P}$ and no $\mathrm{P}$ conditions. This means higher compaction with increasing loads on the samples. Consistence limits and compression index were higher in the P-added samples. Although the optimum moisture for maximum compression index of the surfacelayer was within the plasticity rangeat the surfacelayer sample, which means lower risk to compaction, thesoil would still besusceptibleto compaction becausethesevalues werewithin thefriability range at the 0.27-0.30 m layer sample, which is thedepth affected by most of theplowing practices.
\end{abstract}

Index terms: plasticity, friability, soil compaction, particle dispersion, phosphate sorption.

\section{INTRODUÇÃO}

Nos últimos anos, tem sido observado um aumento expressivo do emprego da motomecanização na agricultura em decorrência da necessidade de maior produtividade. É reconhecida a capacidade desta em criar uma condição de estrutura do solo favorável ao crescimento das plantas, mesmo sob condições edáficas e climáticas desfavoráveis. No entanto, esta vantagem inicial tem sido comprometida pela compactação do solo, atribuído ao tráfego de máquinas e equi pamentos agrícolas (Ribeiro, 1987; Maziero, 1993), principalmente em condições de umidade inadequada para oseu preparo ou uso(Dias J unior, 1994).

Alguns pesquisadores (Ribeiro, 1987; Ballestero, 1992; Maziero,1993) têm investigado as alterações das propriedades físicas do sol o relacionando-as com o uso das máquinas e equipamentos agrícolas. Outros autores têm modelado estas alterações, tentando compreender o processo de compactação, com base, principalmente, na curva de compressão do solo (Figura 1), que representa graficamente a relação entre o logaritmo da pressão aplicada ea densidade do solo (Larson et al., 1980; Larson \& Gupta, 1980; Dias J unior, 1994; Dias J unior \& Pierce, 1996). Esta curva éobti da a partir de um ensai o de compressi bilidade, que consiste na aplicação de pressões sucessivas e contínuas, previamente estabel ecidas, a uma amostra de material de sol o indeformado, em condição parcialmente saturada (Dias J unior \& Pierce, 1996).

A partir da curva de compressão, além da pressão de preconsolidação, é possível determinar o índice de compressão (m). Este índice é representado pela inclinação da reta de compressão virgem (Bradford \& Gupta, 1986), sendotanto maior quanto maior for a inclinação dessa reta, resultando, portanto, em maior susceptibilidade do solo à compactação.

Recentemente, Kondo \& Dias J unior (1999), estudando o efeito do manejo e da umidade no comportamento compressivo de três Latossolos, observaram que o limite de plasticidade (LP) pode representar a umidade que propicia maior índice de compressão (m). Tal observação sugere que o limite de plasticidade (LP) possa indicar a umidade acima da qual o solo não deve ser trabal hado, dado o risco de compactação, pois este limite indica o início da faixa de plasticidade, isto é, faixa onde ocorrem deformações plásticas não-recuperáveis (Hillel, 1982; Dias J unior, 1994).

Na maioria dos trabalhos, o enfoque tem sido predominantemente físico. Todavia, com o desenvolvimento da agricultura tecnificada, o uso de corretivos efertilizantes, principal mentefosfatados, tem sido intensivo.

No caso de solos tropicais, de características oxídicas, onde é verificada grande adsorção de fósforo, é necessária a utilização de grandes quantidades desse macronutriente para suprir a nutrição das plantas. Os benefícios dessa prática são inegáveis, porém, tem sido verificado um efeito dispersivo nos colóides do solo proporcionado pela adsorção de fosfato (Hingston et al ., 1974; Sawhney, 1974; Sposito 1989; Lima et al., 2000). Este efeito pode alterar as propriedades físicas e comprometer o estado de agregação do solo, tornando-o mais susceptível à compactação. I sso ressalta a importância 
da interdependência das propriedades físicas do solo e reações químicas de superfície de partículas no processo de compactação do solo.

Assim, aspectos químicos relacionados com balanço de cargas podem alterar a estrutura do solo, influenciar as propriedades físicas do solo, como os limites de consistência, e, por conseguinte, o seu processo compressivo. Estetipo de estudo ainda não é contemplado na literatura.

Os óxidos de $\mathrm{Fe}$ e $\mathrm{Al}$ são os componentes mais efetivos na adsorção do fosfato (Resende, 1976; Bahia Filho, 1982; Curi, 1983). Segundo esses autores, a magnitude desta adsor ção depende da participação da matéria orgânica, tipos de argila, grau de cristalização, teores e tipos de óxidos e hidróxidos de Fe eAl presentes.

A maior parte das reações envolvendo óxidos e hidróxidos de $\mathrm{Fe}$ e $\mathrm{Al}$, com geração de cargas, é dependente de $\mathrm{pH}$. O predomínio de uma carga na superfície das partículas, positiva ou negativa, produz forças repulsivas entre partículas, aumentando a dispersão e dimi nuindo a estabilidade dos agregados. Em solos oxídicos el etronegativos, a adsorção de $\mathrm{P}$ aumenta a dispersão de partículas, diferentemente dos eletropositivos, nos quais a adsorção de P em um primeiro momento proporciona aumento da floculaçãoe, posteriormente, quando ele se torna el etronegativo, proporciona a dispersão (Hingston et al., 1974; Sawhney, 1974; Sposito, 1989; Lima et al., 2000).

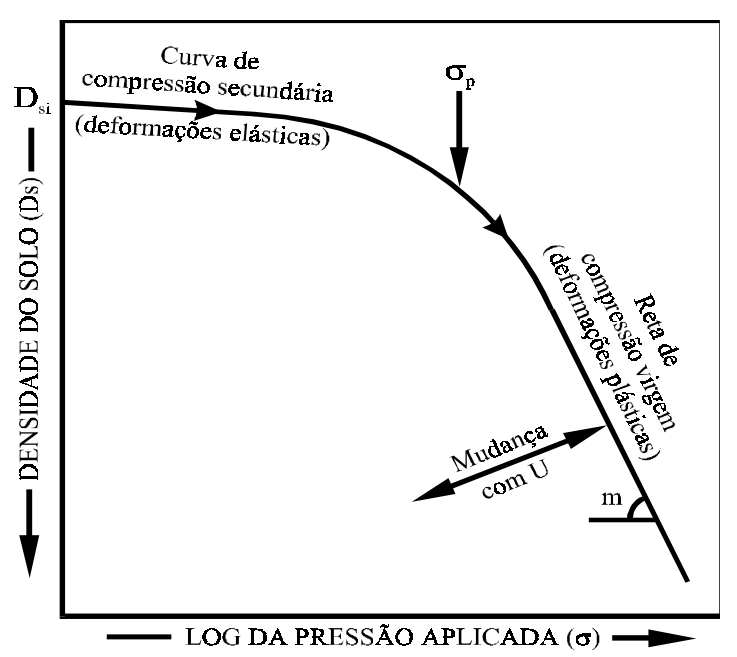

Figura 1. Curva de compressão do solo (DiasJ unior, 1994), mostrando a curva de compressão secundária, reta de compressão virgem com suas respectivas regiões de deformações elásticas e plásticas ( $\sigma_{\mathrm{p}}$ é a pressão de preconsolidação, $\sigma_{c}$ é a pressão crítica, $m$ é o índice de compressão, $U$ é a umidade do solo e $D_{s_{i}}$ é a densidade do solo inicial).
Como a adsorção de fosfato em solos oxídicos proporciona alteração no balanço de cargas, influenciando a floculação e a dispersão de partículas, esta pode também aumentar a susceptibilidade do sol oà compactação, uma vez que as partículas dispersas podem ocupar menores espaços no solo, além da maior exposição da superfície à interação com a água. Com isso, além da umidade, textura, estrutura, história de tensão e densidade do sol o, tal como apresentado na literatura (Dias J unior, 1994; Dias J unior \& Pierce, 1995; Dias J unior \& Pierce, 1996), o manejo, por meio da adubação fosfatada, também podeinfluir nos limites de consistência e no índice de compressão do solo (Silva et al., 1999).

Este estudo teve como objetivo avaliar o efeito da adsorção de fósforo nos índices de compressão (m) e nos parâmetros de consistência em um Latossolo Vermelho-Escuro da microrregião dos Campos da Mantiqueira (MG), Brasil.

\section{MATERIAL E MÉTODOS}

Amostras deformadas e indeformadas $(0,0652 \mathrm{x}$ 0,0254 m de diâmetro e altura, respectivamente) foram coletadas às profundidades de 0-0,03 e 0,270,30 m em um LatossoloVermel ho-E scuro distrófico ( $\mathrm{kr}$ variando de 0,56 a 0,57 e teor de $\mathrm{Fe}_{2} \mathrm{O}_{3}$ de 156 a $163 \mathrm{~g} \mathrm{~kg}^{-1}$, tratando-se, portanto, de um solo oxídico). O solo está localizado nos Campos da Mantiqueira (MG), entre os paralelos $21^{\circ} 15^{\prime}$ e $21^{\circ} 50^{\prime}$ de latitude sul e os meridianos $44^{\circ} 15^{\prime}$ e $44^{\circ} 45^{\prime}$ de longitude, a oeste de Greenwich. Metade das amostras recebeu fósforo (fonte: $\mathrm{KH}_{2} \mathrm{PO}_{4}$ ) suficiente para se atingir a capacidade máxima de adsorção, durante as etapas de pré-umedecimento ou de saturação, conforme o parâmetro avaliado.

\section{Caracterização química e mineralógica}

As amostras deformadas foram submetidas à caracterização química e mineralógica, cujos resultados estão apresentados no quadro 1 . Os óxidos extraídos pelo ataque sulfúrico, fósforo disponível, carbono orgânico e pH foram determinados segundo EMBRAPA (1997). Os teores de caulinita e gibbsita foram quantificados por meio de análise térmica diferencial (ATD), após desferrificação da argila pelo método do ditionito-citrato-bicarbonato (DCB), conforme Mehra \& J ackson (1960). A capacidade máxima deadsorção de fósforo (CMAP) foi calculada com base no $\mathrm{P}$ remanescente (Alvarez, V. \& Fonseca, 1990), seguindo o procedimento metodológico de Olsen \& Watanabe (1957). Os valores do ponto de carga zero (PCZ) foram obtidos indiretamente pela expressão $2 \mathrm{pH}_{\mathrm{KCl}}-\mathrm{pH}_{\mathrm{H}_{2} \mathrm{O}}$, sugerida por Keng (1974). 
Quadro 1. Caracterização química e mineralógica de um Latossolo Vermelho-Escuro da microrregião dos Campos da Mantiqueira (MG)

\begin{tabular}{|c|c|c|c|c|c|c|c|c|c|c|c|c|c|c|c|c|}
\hline \multirow{3}{*}{ Camada } & \multicolumn{14}{|c|}{ Caracterização química(1) } & \multirow{2}{*}{\multicolumn{2}{|c|}{$\begin{array}{l}\text { Caracterização } \\
\text { mineralógica(1) }\end{array}$}} \\
\hline & \multicolumn{5}{|c|}{ Ataque sulfúrico(1) } & \multirow{2}{*}{$\mathbf{F e}$} & \multirow{2}{*}{$\mathbf{F e}_{0}$} & \multicolumn{2}{|c|}{ Rel. molec. } & \multirow{2}{*}{ M.O.(2) } & \multicolumn{2}{|c|}{ pH } & \multirow{2}{*}{$\mathbf{P C Z}^{(3)}$} & \multirow{2}{*}{ CMAP(4) } & & \\
\hline & $\mathrm{SiO}_{2}$ & $\mathrm{Al}_{2} \mathrm{O}_{3}$ & $\mathrm{Fe}_{2} \mathrm{O}_{3}$ & $\mathrm{TiO}_{2}$ & $\mathrm{P}_{2} \mathrm{O}_{5}$ & & & $\mathbf{K i}$ & $\mathrm{Kr}$ & & $\mathrm{H}_{2} \mathbf{O}$ & $\mathrm{KCl}$ & & & $\mathbf{C t} \mathbf{t}^{(5)}$ & $\mathbf{G b}(6)$ \\
\hline $\mathrm{m}$ & - & 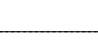 & $-\mathrm{g}$ & $\mathrm{kg}^{-1}$ & 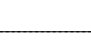 & 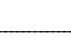 & - & & & $\mathrm{g} \mathrm{kg}^{-1}$ & & & & $\mathrm{mg} \mathrm{kg}^{-1}$ & $-\mathrm{g}$ & $1^{-1}$ \\
\hline $0-0,03$ & 136 & 316 & 156 & 6,9 & 1,02 & 63 & 1,9 & 0,73 & 0,56 & 38 & 5,2 & 4,4 & 3,6 & 1.299 & 240 & 400 \\
\hline $0,27-0,30$ & 137 & 306 & 163 & 8,5 & 0,81 & 59 & 1,6 & 0,76 & 0,57 & 29 & 5,3 & 4,6 & 3,9 & 1.559 & 250 & 380 \\
\hline
\end{tabular}

(1) Média de três repetições. ${ }^{(2)}$ Matéria orgânica. ${ }^{(3)}$ Ponto de carga zero. ${ }^{(4)}$ Capacidade máxima de adsorção de fósforo. ${ }^{(5)}$ Caulinita. (6) Gibbsita.

\section{Caracterização física}

A textura foi avaliada com base no método de Gee \& Bauder (1986). N os tratamentos da condição com fósforo, empregou-se fosfato em concentrações equivalentes à CMAP para $10 \mathrm{~g}$ deTFSE da amostra. O índice de floculação das partículas de argila (IF) foi cal culado com base nas expressões: (a) amostras sem fósforo: IF $=[(A T-A D A) / A T] * 1000$; e (b) amostras com fósforo: IF $F_{P}=[(A T-A D P) / A T] * 1000$; em que IF é oíndice de floculação sem fósforo; I $F_{p}$ é o índice de floculação com fósforo; AT é o teor de argila total, ADA é o teor de argila dispersa em água eADP oteor deargila dispersa em solução defosfato. As quantidades de macro e microporos foram determinadas em amostras indeformadas na presença e ausência de fósforo, conforme método da EMBRAPA (1997). Dados da caracterização física na presença e ausência de fósforo estão apresentados no quadro 2.
Os limites de liquidez (LL) ede plasticidade (LP) foram calculados por meio do método de Sowers (1965) e o limite de contração (LC) de acordo com Bowles (1986). A faixa de friabilidade foi obtida pela diferença entre LP e LC, e a faixa de plasticidade pela diferença entre LL e LP.

\section{Ensaios de compressibilidade}

O índice de compressão $(m)$ de cada amostra foi obtido em ensaios de compressibilidade uniaxial. Nestes ensaios, foram usados corpos de prova de $0,0652 \mathrm{~m}$ de diâmetro por $0,0254 \mathrm{~m}$ de altura com estrutura indeformada, coletados nas camadas de $0-0,03$ e $0,27-0,30 \mathrm{~m}$. Metade das amostras foi saturada por capilaridade em água destilada e metade em solução de fósforo, que continha $\mathrm{P}$ suficiente para se atingir a capacidade máxima de adsorção de fósforo.

Quadro 2. Quantidades de micro e macroporos, argila dispersa, índice de floculação e densidade inicial das amostras nas camadas de 0-0,03 e 0,27-0,30 m de um Latossolo Vermel ho-E scuro da mi crorregião dos Campos da Mantiqueira (MG), nas condições com e sem fósforo. Parâmetros seguidos de letras minúsculas diferentes dentro de cada camada são significativamente diferentes pelo teste de Tukey a $5 \%$

\begin{tabular}{|c|c|c|c|c|c|c|c|c|c|c|c|}
\hline \multirow{3}{*}{ Camada } & \multirow{2}{*}{\multicolumn{3}{|c|}{ Teor de argila }} & \multirow{2}{*}{\multicolumn{2}{|c|}{$\begin{array}{c}\text { Índice de } \\
\text { floculação }\end{array}$}} & \multirow{2}{*}{\multicolumn{2}{|c|}{ Densidade inicial }} & \multicolumn{4}{|c|}{ Porosidade } \\
\hline & & & & & & & & \multicolumn{2}{|c|}{ Macro } & \multicolumn{2}{|c|}{ Micro } \\
\hline & Total (1) & Água(2) & $\mathbf{P}(3)$ & Água & $\mathbf{P}$ & Sem P & Com P & Sem P & Com P & Sem P & Com P \\
\hline $\mathrm{m}$ & 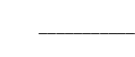 & - & $\mathrm{kg}^{-1}$ & 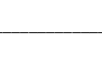 & - & $\longrightarrow M$ & $\mathrm{~m}^{-3}$ & 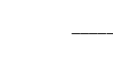 & $\mathrm{m}$ & $m^{-3}$ & - \\
\hline $0-0,03$ & 663 & 150 & 320 & $773 a$ & $517 b$ & $1,01 \pm 0,04$ & $0,99 \pm 0,04$ & $0,222 a$ & $0,184 \mathrm{~b}$ & $0,381 \mathrm{~b}$ & $0,415 a$ \\
\hline $0,27-0,30$ & 685 & 185 & 410 & $729 a$ & $401 \mathrm{~b}$ & $1,06 \pm 0,02$ & $1,05 \pm 0,04$ & $0,232 a$ & $0,210 b$ & $0,386 b$ & $0,411 a$ \\
\hline
\end{tabular}

(1) Teor de argila dispersa em solução hidróxido de sódio. ${ }^{(2)}$ Teor de argila dispersa em água. ${ }^{(3)}$ Teor de argila dispersa em solução de fósforo. Letras minúsculas, na linha, comparam índice de floculação e porosidade na condição com e sem P, a 5\% pel o teste t. 
Após $48 \mathrm{~h}$, as amostras foram transferidas para - extrator de placa porosa para equilibrar os níveis de umidade às faixas de sucção de 2,10 e $1.500 \mathrm{kPa}$. As umidades gravimétricas de 0,25 e $0,05 \mathrm{~kg} \mathrm{~kg}^{-1}$ foram equilibradas à temperatura ambiente.

Em seguida, as amostras foram submetidas aos ensaios de compressibilidade uniaxial, segundo proposta de Dias J unior (1994), usando um consolidômetro da marca Boart Longyear. Cada nível de pressão foi aplicado até que $90 \%$ da máxima deformação do corpo de prova fosseal cançada (Holtz $\&$ Kovacs, 1981). Pressões sucessivas foram aplicadas na seguinte ordem: 25, 50, 100, 200, 400, 800 e $1.600 \mathrm{kPa}$. O índice de compressão, estimado como sendo a inclinação da reta de compressão virgem (Bradford \& Gupta, 1986), como mostrado na figura 1, foi ajustado, considerandoa umidade do sol o (U), à equação polinomial de segunda ordem, sugerida por Dias J unior (1994):

$$
\mathrm{m}=\mathrm{aU}^{2}+\mathrm{bU}+\mathrm{c},
$$

em que

$$
\begin{aligned}
& \mathrm{m}=\text { índice de compressão }\left(\mathrm{Mg} \mathrm{m}^{-3}\right) \\
& \mathrm{U}=\text { umi dade gravimétrica }\left(\mathrm{kg} \mathrm{kg}^{-1}\right) \\
& \mathrm{a}, \mathrm{b}, \mathrm{c} \text { : parâmetros de ajuste }
\end{aligned}
$$

\section{RESULTADOS E DISCUSSÃO}

Os parâmetros de ajuste das equações do índice decompressão $(\mathrm{m})$ com os seus respectivos coeficientes de determinação estão apresentados no quadro 3. Os coeficientes de determinação $\left(R^{2}\right)$ variaram de 0,52 a 0,82 . Tais valores corroboram a aplicabilidade da equação polinomial de segunda ordem para relacionar a umidade e índice de compressão, variável independentee dependente, respectivamente. Resultados semel hantes foram obtidos por Dias J unior (1994), K ondo \& Dias J unior (1999).
Em ambas as camadas e condições estudadas (Figuras 2a, 2b, 3a, 3b), observou-se uma tendência de aumento doíndice de compressão com o conteúdo d'água, até atingir um ponto de máximo, a partir do qual voltou a diminuir, mostrando existir uma umidade ótima para compressão máxima da amostra. Este comportamento condiz com o ajuste proposto por Dias J unior (1994), que está em concordância com Hillel (1982). Na prática, tal informação, aliada ao conhecimento dos limites de consistência, permite inferências a respeito da umidade na qual o solo deva ser adequadamente trabalhado, evitando, assim, a compactação.

A presença de fósforo promoveu um aumento nos valores de $\mathrm{m}_{\text {máximo }}$ (Quadro 4 e Figuras 2a e 2b). A maior expressão desse efeito se deu na camada de 0,27-0,30 m (Figura 2b). Esta alteração foi conseqüência da maior dispersão de partículas, ocasionada, provavelmente, pelo rompimento do efeito cimentante dos óxidos (Shainberg \& Letey, 1984), decorrente da al teração no bal anço de cargas na superfície das partículas. Esta maior dispersão é mostrada pela redução no índice de floculação (Quadro 2) e está em consonância com o menor teor de matéria orgânica e com a mineralogia mais oxídica, que favoreceu maior adsorção de fosfato (Quadro 1).

Vale ressaltar que, neste solo, os val ores de PCZ mostraram-se abaixo dos valores de pH (Quadro 1), o que Ihe conferiu um balanço de carga negativo. Portanto, a adsorção de fosfato intensificou a dispersão de partículas (Lima et al ., 2000), facilitando o preenchimento dos poros (Quadro 2), o que favoreceu maior empacotamento da estrutura no ato da aplicação das cargas durante o ensaio uniaxial.

A menor adsorção de $P$ e, conseqüentemente, o menor efeito dessa adsorção sobre o índice de compressão (m), na camada superficial (Figura 2a), deveu-se ao teor de matéria orgânica ligeiramente superior (Quadro 1) que, de acordo com Afif et al. (1995), foi limitante a essa adsorção (Quadro 1),

Quadro 3. Parâmetros de ajuste das equações de regressões obtidos a partir da relação entre o índice de compressão $(\mathrm{m})$ e a umidade (U), nas camadas de 0-0,03 e 0,27-0,30 m de um Latossolo VermelhoEscuro, nas condições com e sem fósforo.

\begin{tabular}{cccccc}
\hline \multirow{2}{*}{ Camada } & Condição & \multicolumn{3}{c}{ Parâmetro de ajuste da equação } & \multirow{2}{*}{$\mathbf{R}^{\mathbf{2}}$} \\
\cline { 3 - 5 } & & $\mathbf{a}$ & $\mathbf{b}$ & $\mathbf{c}$ & \\
\hline $\mathrm{m}$ & & & & & \\
$0-0,03$ & Sem P & $-2,550$ & 1,490 & 0,176 & $0,71^{* *}$ \\
& Com P & $-2,070$ & 1,490 & 0,132 & $0,82^{* *}$ \\
$0,27-0,30$ & Sem P & $-3,070$ & 1,930 & 0,030 & $0,70^{* *}$ \\
& Com P & $-1,530$ & 0,950 & 0,220 & $0,52^{*}$ \\
\hline
\end{tabular}

\footnotetext{
* e** Significativos a 5 e 1\%, respectivamente, pelo teste $\mathrm{F}$.
} 
Quadro 4. Limites de consistência, faixas de friabilidade e plasticidade, índice de compressão máximo $\left(m_{\text {máx }}\right)$ com respectivas umidades óti mas $\left(U_{\text {ótima }}\right)$, nas camadas de 0-0,03 e 0,27-0,30 $m$ de um Latossolo Vermelho-E scuro, nas condições com e sem fósforo

\begin{tabular}{|c|c|c|c|c|c|c|c|c|}
\hline \multirow{2}{*}{ Camada } & \multirow{2}{*}{ Condição } & \multicolumn{3}{|c|}{ Limite de consistência } & \multirow{2}{*}{$\begin{array}{l}\text { Faixa de } \\
\text { friabilidade }\end{array}$} & \multirow{2}{*}{$\begin{array}{l}\text { Faixa de } \\
\text { plasticidade }\end{array}$} & \multirow{2}{*}{ U máxima } & \multirow[b]{2}{*}{ mmáximo } \\
\hline & & LL & LP & LC & & & & \\
\hline $\mathrm{m}$ & & 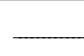 & - & 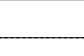 & $-\mathrm{kg} \mathrm{kg}^{-1}$ & 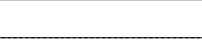 & L & $\mathrm{Mg} \mathrm{m}^{-3}$ \\
\hline $0-0,03$ & $\begin{array}{l}\text { Sem fósforo } \\
\text { Com fósforo }\end{array}$ & $\begin{array}{l}0,44 \\
0,62\end{array}$ & $\begin{array}{l}0,33 \\
0,39\end{array}$ & $\begin{array}{l}0,22 \\
0,26\end{array}$ & $\begin{array}{l}0,11 \\
0,13\end{array}$ & $\begin{array}{l}0,11 \\
0,23\end{array}$ & $\begin{array}{l}0,28 \\
0,37\end{array}$ & $\begin{array}{l}0,39 \\
0,40\end{array}$ \\
\hline $0,27-0,30$ & $\begin{array}{l}\text { Sem fósforo } \\
\text { Com fósforo }\end{array}$ & $\begin{array}{l}0,40 \\
0,57\end{array}$ & $\begin{array}{l}0,25 \\
0,35\end{array}$ & $\begin{array}{l}0,16 \\
0,22\end{array}$ & $\begin{array}{l}0,09 \\
0,13\end{array}$ & $\begin{array}{l}0,15 \\
0,22\end{array}$ & $\begin{array}{l}0,31 \\
0,31\end{array}$ & $\begin{array}{l}0,33 \\
0,37\end{array}$ \\
\hline
\end{tabular}

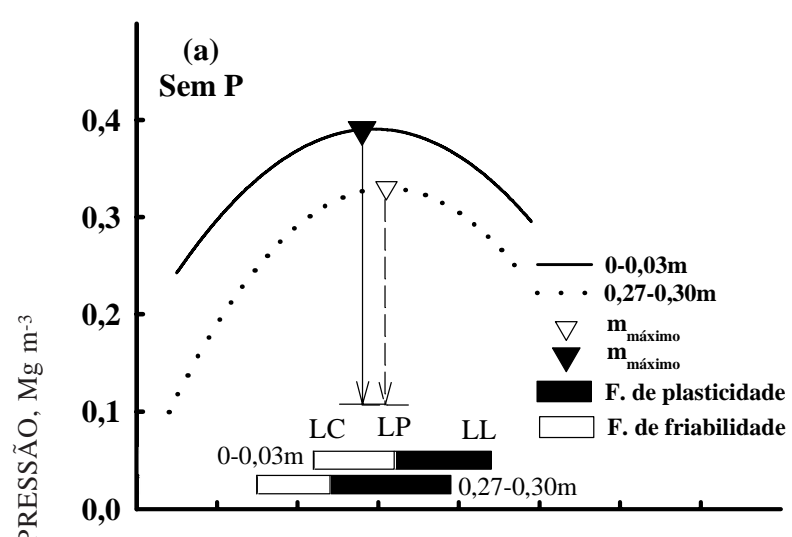

(b)

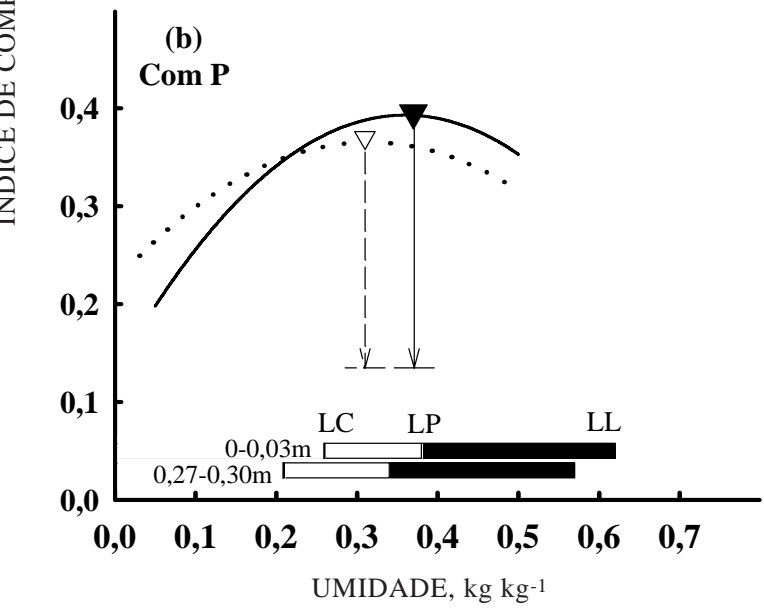

Figura 2. Índice de compressão $(m)$ em função da umidade $(U)$, nas condições com e sem $P$, nas camadas de 0-0,03 e 0,27-0,30 m de um Latossolo Vermelho-E scuro.

principalmente por competir com oíon fosfato pelos síti os de adsor ção da superfície dos óxidos. Contudo, embora a camada de 0,27-0,30 m tenha apresentado maior resposta à compressão, em virtude da aplicação de fósforo, foi na camada de 0-0,03 m que se observou maior valor de índice de compressão máximo (mmáx), independentemente da aplicação de fósforo (Figura 3a e 3b). Tal fato deveu-se à contribuição mais efetiva da matéria orgânica na estrutura dessa camada, que Ihe conferiu menores val ores de densi dade do sol o inicial (Dsi ) (Quadro 2) e mai or deformação durante a aplicação das cargas. Este comportamento compressivo conferiu a essa camada menor suporte de carga e maior susceptibilidade à compactação, principalmente se trabalhada em umidades inadequadas (Dias J unior, 1994; Kondo \& Dias J unior, 1999; Silva et al., 1999).

Ainda com base na figura 3 , observou-se 0 desl ocamento dos valores dos limites de consistência para umidades mais el evadas (Figura 3a, 3b). Este deslocamento conferiu diferentes faixas de friabilidadee plasticidade, mostrando quea umidade adequada de preparo do solo de uma camada não se aplica a outra. Outro aspecto por ressaltar foi o deslocamento do $m_{\text {máx }}$ na de camada $0-0,03 \mathrm{~m}$ no sentido da faixa de plasticidade (Figura 3a), o que, para o manejo, é positivo, já que se ganha maior faixa de umidade para trabalhabilidade, dada a possibilidade de a compressão máxima do solo deslocar-se para umidades mais elevadas. $\mathrm{Na}$ camada de 0,27-0,30 m (Figura 3b), embora as umidades ótimas ( $\left.U_{\text {ótima }}\right)$ tenham sido coincidentes, o $m_{\text {máx }}$, na condição com fósforo encontrou-sena faixa de friabilidade, fato que, certamente, revela maior cuidado durante o preparo dessesolo, visto que, nessa camada, de modo geral, encontra-sea profundidade de corte de al guns implementos agrícolas.

Os limites deconsistência na condição com fósforo (Figura 3a e 3b) deslocaram-se para valores mais el evados de umidade. Esse deslocamento foi provocado pela adsorção de $\mathrm{P}$, em decorrência dos teores de óxidos, que intensificou a dispersão, aumentando a quantidade de microporos e, conseqüentemente, proporcionou maior retenção de água no solo. 


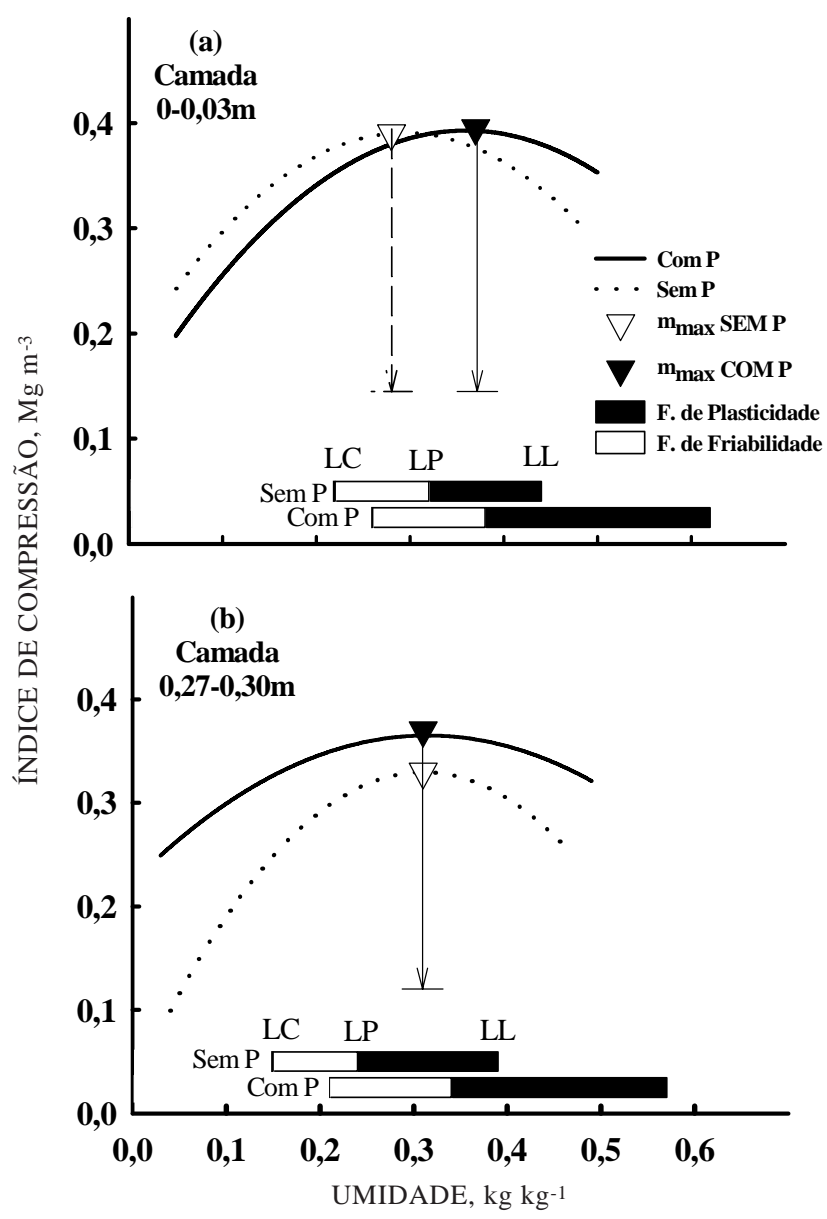

Figura 3. Índice de compressão (m) em função da umidade (U), nas camadas de 0-0,03 e 0,270,30 m de um Latossolo Vermelho-E scuro, na condição com e sem $P$.

\section{CONCLUSÕES}

1. Materiais de solo da camada de 0-0,03 m apresentaram maiores índices de compressão (m), sendo, portanto, mais susceptíveis à compactação do que materiais da camada de 0,27-0,30 m.

2. A adição de fósforo proporcionou um deslocamento dos limites de consistência do Latossol o Vermelho-E scuro para valores mais elevados de umidade e promoveu um aumento nos índices de compressão $(\mathrm{m})$, tornando este solo mais susceptível à compactação.

3. Embora $m_{\text {máx }}$ de materiais de solo da camada de 0-0,03 m do LatossoloVermelho-Escuro encontrese na faixa de plasticidade, cuidado deve ser tomadose durante o preparo desse solo, já que o $\mathrm{m}_{\text {máx }}$ da camada de 0,27-0,30 m, profundidade de corte de alguns implementos agrícolas, foi deslocado para a faixa de friabilidade.

\section{LITERATURA CITADA}

AFIF, E.; BARRON, V. \& TORRENT, J . Organic matter delays but does not prevent phosphate sorption by cerrado soil from Brazil. Soil Sci., 59:207-211, 1995.

ALVAREZV.,V.H. \& FONSECA, D.M. Definição de doses de fósforo para a determinação da capacidade máxima de adsorção de fosfatos e para ensaios em casa de vegetação. R. Bras. Ci. Solo, 14:49-55, 1990

BAHIA FILHO, A.F.C. Índice de disponibilidade de fósforo em Latossolos do Planalto Central com diferentes características texturais e mineralógicas. Viçosa, Universidade Federal de Viçosa, 1982. 179p. (Tese de Doutorado)

BALLESTERO, S.D. Efeito da semeadura de milho em quatro níveis de umidade na compactação de um Latossolo Vermelho-Escuro. Piracicaba, Escola Superior deAgricultura Luiz de Queiroz, 1992. 89p. (Tese de Doutorado).

BOWLES, J.A. Engineering properties of soils and their measurements. 3.ed. New York, McGraw-Hill, 1986. 218p.

BRADF ORD, J .M. \& GUPTA, S.C. Compressibility. In: KLUTE, A., ed. Methods of soil analysis. Part 1. 2 ed. Madison, American Society of Agronomy, 1986. p.279-492.

CURI, N.; CAMARGO, O.A.; GUEDES G.A.A. \& SILVEIRA, J.V. Sorção de fósforo em materiais de Latossolo do Brasil Sudeste. In: REUNIÃO DE CLASSIFICAÇÃO, CORRELAÇÃO DE SOLOS E INTERPRETAÇÃO DA APTIDÃO AGRÍ COLA, 3., Rio de J aneiro, 1988. Anais. Rio de J aneiro, Empresa Brasileira de Pesquisa Agropecuária, 1988. p.267-282.

DIAS J UNIOR, M.S. \& PIERCE, F.J . O processo de compactação do sol o e sua model agem. R. Bras. Ci. Sol o, 20:175-182, 1996.

DIAS J UNIOR, M.S. \& PIERCE, F.J . A simple procedure for estimating preconsolidation pressurefrom soil compression curves. Soil Technol ., 8:139-151, 1995.

DIAS J UNIOR, M.S. Compression of three soils under long-term tillage and wheel traffic. East Lansing, Michigan State University, 1994. 114p. (Tese de Doutorado)

EMPRESA BRASILEIRA DE PESQUISA AGROPECUÁRIA EMBRAPA. Centro Nacional de Pesquisa de Solos. Manual de métodos de análise de solo. Rio de J aneiro, 1997. 212p.

GEE, G.W. \& BAUDER, J.W. Particle size analysis. KLUTE, A., ed. Methods of soils analysis. 2.ed. Madison, American Society of Agronomy, 1986. pt.1. p.383-411. (Agronomy Monography, 9)

HILLEL, D. Introduction to soil physics. San Diego, Academic Press, 1982. 364p.

HINGSTON, F.J .; POSNER A.M. \& QUIRK.J .P. Anion adsorption by goethite and gibbsite. II: Desorption of anions from the hydrous oxide surfaces. J. Soil Sci., 25:16-26, 1974.

HOLTZ, R.D. \& KOVACS, W.D. Introduction to geotechnical engineering. Englewood Cliffs, Prentice-Hall, 1981. 773p.

KENG,J.C. \& UEHARA, G. Chemistry, mineralogy and taxonomy of oxissols and Utisols. Proc. Soil Crop Sci. Soc., 33:119126, 1974.

KONDO, M.K. \& DIAS J UNIOR, M.S. Efeito do manejo e da umidade no comportamento compressivo de três Latossol os. R. Bras. Ci. Solo, 23:497-506, 1999. 
LARSON, W.E. \& GUPTA, S.C. Estimating critical stress in unsaturated soil from changes in pore water pressure during confined compression. Soil Sci. Soc. Am. J ., 44:1127$1132,1980$.

LARSON, W.E.; GUPTA, S.C. \& USECHE, R.A. Compression of agricultural soils from eight soil orders. Soil Sci. Soc. Am. J., 44:450-457, 1980.

LIMA, J.M.; ANDERSON, S.J . \& CURI, N. Phosphate-induced clay dispersion as related to aggregate size and composition in hapludoxs. Soil Sci. Soc. Am. J., 64:892-897, 2000.

MAZIERO, J.V.G. Compactação do solo por patinagem da roda motriz de um trator agrícola. Piracicaba, Escola Superior de Agricultura Luiz de Queiroz, 1993. 105p. (Tese de Mestrado)

MEHRA, O.P. \& J ACKSON, M.L. I ron oxides removal from soils and clays by a dithionite-citrate-bicarbonate system buffered with sodium bicarbonate. Clays Clay Miner., 7:317327, 1960.

OLSEN, R.B. \& WATANABE, F.S. A method of determine a phosphorus adsorption maximum of soils as measured by the Langmuir isotherm. Soil Sci. Soc. Am. J., 21:144-149, 1957.
RESENDE, M. Mineralogy, chemistry, morphology and geomorphology of some soils of the Central Plateau of Brazil. West Lafayette, Purdue University, 1976. 237p (Tese de Doutorado)

RIBEIRO, S.A. Efeitos da col heita mecânica na produção de capim el efante (Pennisetum purpureum, Schum) Piracicaba, Escola Superior de Agricultura Luiz de Queiroz, 1987. 64p. (Tese de Mestrado)

SAWHNEY, B.L. Charge characteristics of soils as affected by phosphate sorption. Soil Sci. Soc. Am. J ., 38:159-160, 1974.

SHAINBERG, I \& \& LETEY, J. Response of soil to sodic and saline conditions. J. Agric. Sci., 52:1-57, 1984.

SILVA, R.B.; LI MA,J .M. \& DIAS J UNIOR, M.S. Efeito da adsorção de fosfato em parâmetros físicos e na compressibilidade de solos tropicais. R. Bras. Ci. Solo, 23:219-226, 1999.

SOWERS, G.W. Consistency. In: KLUTE, A., ed. Methods of soil analysis. Part 1. 2.ed. Madison, American Society of Agronomy, 1965. p.391-399 (Agronomy Monography)

SPOSITO, G. Surface reactions in natural and aqueous colloidal systems. Chimia, 43:169-176, 1989. 